\title{
Analisi dell'evoluzione degli strumenti internazionali volti al contrasto della violenza di genere
}

\section{Analysis of the evolution of international instruments aimed at combating gender violence}

Agostina Latino*

\section{Riassunto}

II fenomeno della violenza nei confronti delle donne è una vera e propria pandemia che non conosce confini poiché investe indistintamente tutto il mondo, sia pure con declinazioni e tassi di incidenza diversi. La violenza nei confronti delle donne assume diverse forme, alcune più frequenti di altre, nei diversi Paesi del mondo. La Convention on the Elimination of All Forms of Discrimination against Women - CEDAW, all'art. 17 - Parte V, prevede l'istituzione di un "Comitato per l'eliminazione della discriminazione nei confronti della donna" che ha il compito di sorvegliare lo stato di applicazione delle norme da parte degli Stati contraenti. La Convenzione sulla prevenzione e la lotta contro la violenza nei confronti delle donne e la violenza domestica è stata adottata quindi dal Comitato dei Ministri del Consiglio d'Europa il 7 aprile 2011 ed è stata aperta alla firma l'11 maggio 2011 in occasione della $121^{\mathrm{ma}}$ sessione del Comitato dei Ministri a Istanbul. La Convenzione di Istanbul rappresenta sicuramente un punto di riferimento a livello internazionale per quanto riguarda la lotta alla violenza contro le donne.

Parole Chiave: Violenza nei confronti delle donne. Strumenti internazionali. Convenzione di Istanbul.

\section{Abstract}

The phenomenon of violence against women is a real pandemic that knows no boundaries because investing indiscriminately around the world, albeit with

Doutora em Direito Internacional pela Università degli Studi di Bari. Professora pesquisadora na Facoltà di Giurisprudenza - Universidade de Camerino. Camerino - Itália. Email: agostina.latino@ unicam.it 
different variations and incidence rates. Violence against women takes many forms, some more common than others, in the various countries of the world. The Convention on the Elimination of All Forms of Discrimination against Women - CEDAW, art. 17 - Part V provides for the establishment of a "Committee for the Elimination of Discrimination against Women" which is responsible for monitoring the conditions of application of rules by the Contracting States. The Convention on preventing and combating violence against women and domestic violence was adopted then by the Committee of Ministers of the Council of Europe 7 April 2011 and was opened for signature on 11 May 2011 at the 121ma session of the Committee of Ministers in Istanbul. The Istanbul Convention is certainly a point of reference at the international level with regard to combating violence against women.

Keywords: Violence against women. International instruments. Istanbul Convention.

\section{Introduzione}

Fin dal 1999, l'Assemblea generale delle Nazioni unite (NU), con la risoluzione n. 54/134, ha proclamato il 25 novembre "Giornata internazionale per l'eliminazione della violenza contro le donne". La data è stata scelta in ricordo del brutale assassinio delle sorelle Mirabal (Patria, Minerva e Maria Teresa) avvenuto nel 1960 nella Repubblica Dominicana, sotto la feroce dittatura di Trujillo. Le tre sorelle, soprannominate "las mariposas", le farfalle, per la loro bellezza e vivacità, il 25 novembre 1960 , furono catturate, torturate, uccise e gettate con la loro auto in un precipizio, al fine di simulare una morte accidentale, da agenti segreti del servizio di informazione militare, mentre si recavano a visitare i loro mariti in prigione per motivi politici.

Il fenomeno della violenza nei confronti delle donne è una vera e propria pandemia che non conosce confini poiché investe indistintamente tutto il mondo, sia pure con declinazioni e tassi di incidenza diversi. 
La violenza nei confronti delle donne è un tema risalente nel tempo ${ }^{1}$. In queste brevi note, dopo aver individuato le forme più diffuse di violenza nei confronti delle donne, cercheremo di ricostruire le tappe degli strumenti adottati sotto l'egida delle Nazioni unite e nei quadri regionali, per analizzare più specificamente la Convenzione di Istanbul, ossia la Convenzione del Consiglio d'Europa sulla prevenzione e la lotta alla violenza contro le donne e la violenza domestica, approvata dal Comitato dei Ministri del Consiglio d'Europa il 7 aprile 2011, aperta alla firma l'11 maggio 2011, entrata in vigore il $1^{\circ}$ agosto 2014(COUNCIL OF EUROPE, online, 2011).

\section{Forme di violenza nei confronti delle donne}

La violenza nei confronti delle donne assume diverse forme, alcune più frequenti di altre, nei diversi Paesi del mondo. Peraltro, un comune denominatore che può essere rilevato nella più gran parte dei casi è la povertà, posto che, secondo i dati NU più del $70 \%$ degli individui che vive al di sotto della soglia di povertà è di sesso femminile. II dilagare della violenza nei confronti delle donne è favorito altresì da normative interne poiché alcuni ordinamenti adottano misure discriminatorie che impediscono la piena parità di diritti fra uomini e donne.

Le modalità più diffuse di violenza nei confronti delle donne sono riconducibili principalmente a cinque schemi: violenza domestica, pedofilia, tratta, mutilazioni genitali, stupro di guerra.

Sotto il primo profilo, può innanzitutto ricordarsi come secondo una ricerca condotta dall'Organizzazione Mondiale della Sanità (WORLD HEALTH ORGANIZATION, online, 2013), il 70\% delle donne vittime di omicidi sono state uccise dai loro partners maschili. L'ampiezza di

\footnotetext{
'nternational Human Rights Treaties. Issues on Rape, Domestic Slavery, Abortion and Domestic Violence, in International Journal of Constitutional Law, 2007, p. 507 ss., e A. DEL VECCHIO, La tutela dei diritti delle donne nelle convenzioni internazionali, in T. VASSALLI DI DACHENHAUSEN (a cura di), Atti del Convegno in memoria di Luigi Sico: il contributo di Luigi Sico agli studi di diritto internazionale e di diritto dell'Unione europea, Napoli, 2011, p. 315 ss.7
} 
questa forma di violenza è del tutto trasversale: colpisce infatti anche i Paesi più evoluti in termini di organizzazione sociale e di garanzia dei diritti individuali. La violenza domestica può assumere diverse forme: comprende violenza fisica, sessuale, psicologica, minacce, intimidazioni, persecuzioni, coercizioni, divieti, segregazione, umiliazioni, violenza economica (come negazione di disponibilità finanziarie, dell'acquisto di vestiario o altro, del cibo, di cure mediche e perfino appropriazione del reddito). I dati, in crescita preoccupante in tutti i Paesi occidentali, non fotografano la totalità dei casi, poiché esiste un sommerso (che secondo alcuni analisti statistici rappresentano la maggior parte) di casi non denunciati dalla vittima, per paura di ripercussioni o per vergogna².

Sotto il secondo profilo, la pedofilia, ossia le molestie sessuali nei confronti di bambine e adolescenti, si registrano dati agghiaccianti per dimensioni. Uno studio dell'United Nations Children's Fund (UNICEF, online, 2014a), che ha analizzato tanto Paesi sviluppati che Paesi in via di sviluppo, indica che tra l' 1 e il $21 \%$ delle donne ha denunciato di essere stata abusata sessualmente prima del $15^{\circ}$ anno di età, nella maggior parte dei casi da membri maschi della famiglia. In particolare, lo studio rileva che una percentuale variante tra il 7 e il $36 \%$ delle donne e il 3 e il $29 \%$ degli uomini afferma d'esser stata vittima di abusi sessuali durante l'infanzia, e, a seconda del Paese analizzato, il tasso di abusi tra le bambine è da una volta e mezzo a tre volte superiore a quello dei bambini, abusi che, nella maggior parte dei casi, avvengono in ambito familiare. Secondo uno studio condotto negli USA, I'83\% delle alunne delle classi dall' $8^{\circ}$ all' $11^{\circ}$ livello (tra i 12 e i 15 anni) che frequentano le scuole pubbliche, subiscono qualche forma di molestia sessuale (AMERICAN ASSOCIATION OF UNIVERSITY WOMEN, online, 2001) (UNITED NATIONS, online, 2006).

\footnotetext{
Per esempio, uno studio basato su interviste somministrate a 42 mila donne dei 28 Paesi membri dell'Unione europea ha rilevato che solo il $14 \%$ delle vittime ha denunciato alle forze dell'ordine le violenze subite dal proprio partner: Violence against Women: an EU-Wide Survey, European Union, 2014, Foreword, p. 3.
} 
Sotto il terzo profilo, la tratta, ci si riferisce al reclutamento e al trasferimento di una persona a fini di sfruttamento (prevalentemente prostituzione). È una declinazione moderna della schiavitù, che è ovviamente, in sé, una violazione dei diritti umani, ma ne implica inevitabilmente altri, quali maltrattamenti, torture, segregazione, malnutrizione, mancanza di cure mediche, fino a giungere, talvolta alla perdita della vita. A livello mondiale, le vittime della tratta di esseri umani sono stimate a due milioni e mezzo all'anno. L'ottanta per cento di loro sono donne e ragazze. Secondo l'Organizzazione Mondiale del Lavoro (International Labour Organization - ILO), sono circa 1,2 milioni i minori di 18 anni vittime di tratta nel mondo (INTERNATIONAL LABOUR ORGANIZATION, online, 2012).

Sotto il terzo profilo, le mutilazioni genitali femminili (MGF), parziali o totali, sono pratiche diffuse soprattutto in buona parte dell'Africa (Somalia, Gibuti, Sudan, Etiopia, Somalia e alcune regioni e/o gruppi di popolazione del Kenya, Nigeria, Mali, Mauritania), in alcuni Paesi del Medio Oriente (Egitto, Oman, Emirati Arabi Uniti) e in qualche zona o comunità dell'Asia (India, Indonesia, Malesia e Sri Lanka). II fenomeno riguarda anche l'Europa relativamente ai migranti provenienti da zone in cui questa pratica è perpetrata. Secondo I'UNICEF le MGF riguardano ogni anno circa tre milioni di bambine o ragazze e sono oggi circa 140 milioni le donne che l'hanno subita (UNICEF, online, 2014b).

Sotto il quarto profilo, lo stupro di guerra, ossia lo stupro di massa di donne da parte delle truppe che hanno invaso un Paese, o, in una guerra interna, da parte di una fazione in lotta contro le donne dell'altra, assume talvolta i connotati della pulizia etnica, poiché la nascita di figli "misti" è una forma di umiliazione su un popolo, che non si vuole solamente vincere militarmente e depredare, ma anche violentare attraverso le sue donne da cui nascono figli dei vincitori. Come esempi di prassi basta ricordare che durante la guerra in Bosnia, dal 1992 al 1995, si stima che siano state violentate 50.000 donne; mentre durante la guerra civile in Ruanda nel 1994 fra Hutu e Tutsi (nel corso della quale fu ucciso circa un milione di Tutsi) furono violentate da 250.000 a 500.000 donne della 
etnia soccombente, di cui più del $67 \%$ ha contratto I'AIDS (AMNESTY INTERNATIONAL, online, 2004).

\section{La tutela delle donne negli strumenti internazionali}

L'Organizzazione delle NU, fin dalla sua nascita, pur riconoscendo l'unitarietà dei diritti umani, ha preso atto delle peculiarità proprie della tutela delle donne, e, a tal fine, il Consiglio di sicurezza (CdS), con la risoluzione 11(II) del 21 giugno 1946, ha istituito la Commissione sullo Status delle Donne (Commission on the Status of Women - CSW), con il mandato di preparare raccomandazioni e rapporti indirizzati al Consiglio Economico e Sociale (Economic and Social Council ECOSOC) sul tema della promozione dei diritti delle donne in ambito politico, economico, civile, sociale ed educativo. Nei primi vent'anni di attività, la Commissione si è dedicata principalmente alla negoziazione di trattati internazionali miranti a modificare le legislazioni discriminatorie ancora in vigore nella maggior parte dei Paesi; ma fin dai primi anni '50 ha prestato particolare attenzione ad alcune specifiche fattispecie di violenza di genere, quali ad esempio le mutilazioni genitali e altre pratiche tradizionali che minacciassero l'integrità fisica e psichica di donne e ragazze. L'impulso propulsivo per la promozione della tutela delle donne è dato dalla I Conferenza Mondiale sulla condizione della donna di Città del Messico (1975), in concomitanza con l'Anno Internazionale delle Donne, che lancia il Decennio delle Nazioni Unite per le Donne (1976-1985). II frutto più rilevante di questo decennio è sicuramente la Convenzione sull'eliminazione di ogni forma di discriminazione contro le donne (UNITED NATIONS, online, 1979), adottata il 18 dicembre 1979 dall'Assemblea generale delle NU, che costituisce un vero e proprio bill of rights dei diritti delle donne. In verità nella CEDAW non si fa mai esplicito riferimento alla violenza di genere come fenomeno specifico, ma si disciplinano fattispecie particolari di violenza, quali, ad esempio, i matrimoni forzati (art.16) e lo sfruttamento sessuale (art. 6). La CEDAW è entrata in vigore il 3 settembre 1981, dopo poco più di due anni dalla sua firma, in tempi record quindi rispetto agli altri Trattati in tema di diritti 
della persona umana. Questo successo è però mitigato dal gran numero di riserve e dichiarazioni interpretative che ne attenuano la portata precettiva. La Convenzione, articolata in un preambolo e 30 articoli, è stata ratificata da 188 Stati, fra cui non figurano Stati Uniti, Città del Vaticano, Iran, Nauru, Palau, Somalia, Sudan, Tonga. I pilastri principali della Convenzione, di stampo prettamente emancipatorio, sono quattro: in primo luogo, sono definite le forme di discriminazioni nei confronti delle donne; in secondo luogo, si impone agli Stati parte di astenersi da azioni discriminanti in base al sesso; in terzo luogo, si ingiunge agli Stati parte di adottare provvedimenti per raggiungere l'uguaglianza uomodonna in tutti i settori (peraltro è previsto che possano essere disposte misure ineguali da applicare temporaneamente, miranti a realizzare l'eguaglianza de facto uomo-donna); in quarto luogo, infine, si garantisce alla donna parità di diritti nella vita pubblica e politica, nell'acquisizione di cittadinanza diversa da quella di nascita, nell'istruzione, nella vita professionale, nel sistema sanitario, nel diritto matrimoniale e in quello di famiglia. La Convenzione, all'art. 17 - Parte V, prevede l'istituzione di un "Comitato per l'eliminazione della discriminazione nei confronti della donna" che ha il compito di sorvegliare lo stato di applicazione delle norme da parte degli Stati contraenti. II Comitato è composto da 23 membri eletti a scrutinio segreto su una lista di candidati designati dagli Stati parte, che hanno l'obbligo di presentare, al Comitato, almeno ogni 4 anni, un rapporto in cui sono illustrate le azioni compiute dallo Stato in questione per dare applicazione alle norme in essa contenute ma anche i fattori e le difficoltà che influiscono sul grado di applicazione degli obblighi previsti dalla presente Convenzione. La presentazione dei rapporti (motu proprio da parte degli Stati contraenti o su invito del Comitato) è "a porte aperte"; il Comitato, inoltre, riceve informazioni in via informale dalle organizzazioni non governative (ONG) le quali possono anche assistere alle sedute del Comitato. Successivamente, a quasi 20 anni dall'entrata in vigore della CEDAW, il 15 ottobre 1999, l'Assemblea generale $(A G)$ delle NU ha adottato un Protocollo facoltativo, entrato in vigore fra gli Stati ratificanti il 22 dicembre 2000, che ha allineato la Convenzione ai principali testi internazionali in materia di diritti umani 
e ha fornito più chiare possibilità di ricorso in caso di violazioni. II Protocollo infatti definisce due diverse procedure di intervento: da un lato, una procedura di denuncia, utilizzabile sia da singole donne che da gruppi di donne per denunciare al Comitato i casi di violazione delle norme stabilite dalla Convenzione; sia una procedura d'indagine, che conferisce al Comitato il potere di condurre indagini sui casi di violazioni gravi o sistematiche dei diritti umani delle donne nei Paesi che hanno sottoscritto il Protocollo facoltativo. Va peraltro ricordato che il Comitato non ha un vero e proprio potere sanzionatorio nei confronti degli Strati parte: l'eventuale riscontro di comportamenti non collimanti con quanto prescritto nella CEDAW comporta solamente un rapporto dal valore di soft law iscrivibile alla moral suasion.

Nel 1985, nel quadro della III Conferenza Mondiale delle Donne (Nairobi) è stato approvato un Piano d'Azione nel quale si prende atto che la violenza nei confronti delle donne non è un problema da confinarsi un ambito eminentemente privato ma, al contrario, è una questione pubblica che - come tale - richiede un approccio proattivo tanto a livello statale quanto a livello internazionale. Ciò ha portato, nel giugno del 1993, alla votazione unanime dei rappresentanti dei 171 Stati presenti alla Conferenza Mondiale delle Nazioni Unite sui Diritti Umani a Vienna, di una Dichiarazione e di un Programma d'Azione per la promozione e la tutela dei diritti umani nel mondo, ponendo l'accento sulla "importanza di lavorare per l'eliminazione della violenza contro le donne nella vita pubblica e privata, per l'eliminazione di tutte le forme di molestie sessuali, sfruttamento e tratta delle donne, per l'eliminazione di pregiudizi di genere nell'amministrazione della giustizia e per lo sradicamento di ogni conflitto che possa insorgere tra i diritti delle donne e gli effetti dannosi di certe pratiche tradizionali o abituali, di pregiudizi culturali ed estremismi religiosi" (art 38). Con la risoluzione 48/104 del 20 dicembre 1993, adottata per consensus (ossia in modo unanime per mancanza di obiezioni senza la necessità di ricorrere al voto palese e nominale), l'Assemblea Generale delle NU ha promulgato la Dichiarazione sull'eliminazione della violenza contro le donne. Il documento contempla la definizione più diffusa di violenza contro le donne: ai sensi dell'art. 1, «l'espressione "violenza 
contro le donne" significa ogni atto di violenza fondata sul genere che abbia come risultato, o che possa probabilmente avere come risultato, un danno o una sofferenza fisica, sessuale o psicologica per le donne, incluse le minacce di tali atti, la coercizione o la privazione arbitraria della libertà, che avvenga nella vita pubblica o privata»; l'art. 2 prosegue affermando che «la violenza contro le donne dovrà comprendere, ma non limitarsi alla violenza fisica, sessuale e psicologica che avviene in famiglia, incluse le percosse, l'abuso sessuale delle bambine nel luogo domestico, la violenza legata alla dote, lo stupro da parte del marito, le mutilazioni genitali femminili e altre pratiche tradizionali dannose per le donne, la violenza non maritale e la violenza legata allo sfruttamento; alla violenza fisica, sessuale e psicologica che avviene all'interno della comunità nel suo complesso, incluso lo stupro, l'abuso sessuale, la molestia sessuale e l'intimidazione sul posto di lavoro, negli istituti educativi e altrove, il traffico delle donne e la prostituzione forzata; alla violenza fisica, sessuale e psicologica perpetrata o condotta dallo Stato, ovunque essa accada».

La violenza sessuale assurge ai vertici dell'agenda mondiale quando, nel corso della guerra nella ex lugoslavia ${ }^{3}$ e nel Rwanda ${ }^{4}$, assume proporzioni di fenomeno di massa: lo stupro viene adoperato

3 Come già ricordato, si stima che durante la guerra in Bosnia Erzegovina circa 50.000 donne sono state violentate. La maggioranza delle vittime delle violenze erano donne musulmane stuprate dai soldati serbi, al fine di obbligare il gruppo etnico oggetto delle violenze ad andarsene dalla regione. Addirittura, è stata documentata l'esistenza di "campi di stupro" creati deliberatamente per ingravidare le donne musulmane e croate tenute prigioniere. Viene documentato inoltre che spesso le donne erano tenute in prigionia fino all'ultima fase della gravidanza, al fine di "creare" una nuova generazione serba (posto che l'etnia si erediti in linea paterna). Del pari, durante la guerra del Kosovo, migliaia di donne kosovare-albanesi sono state vittima di violenze sessuali. (HUMANS RIGHTS WATCH, online, 2001)

4 Nel rapporto del 1996 il Relatore Speciale delle Nazioni Unite in Rwanda, Rene Degni-Segui, affermò che «lo stupro era la regola e la sua assenza l'eccezione». II rapporto afferma inoltre che «lo stupro era sistematico ed era usato come "arma" dai perpetratori del massacro». Un rapporto del 2000 del Gruppo Internazionale di Personalità Eminenti dell'Organizzazione dell'Unità Africana concluse che «possiamo essere certi che tutte le donne che sopravvissero al genocidio furono vittime dirette di stupro o altre violenze sessuali o furono profondamente sconvolte da essi». II Relatore Speciale in Rwanda stimò nel rapporto del 1996 che tra 2.000 e 5.000 gravidanze furono il risultato degli stupri di guerra, e che tra 250.000 e 500.000 donne e ragazze ruandesi furono violentate. Va ricordato che il Rwanda è una società patrilineare e che quindi i bambini prendono l'etnia del padre, il che sottolinea il carattere genocidario dello stupro di massa. (BROUWER, 2005, p. 10) 
come sistemica tattica di guerra, sicché tanto lo Statuto del Tribunale penale internazionale per l'ex Jugoslavia (International Criminal Tribunal for the former Yugoslavia - ICTY), istituito con ris. 827/25.05.1993 del CdS delle NU, quanto lo Statuto del Tribunale per il Rwanda (International Criminal Tribunal for Rwanda - ICTR), istituito con ris. 955/8.11.1994 del CdS delle NU, includono nella propria competenza ratione materiae (anche) crimini quali stupro e violenze sessuali (rispettivamente, all'art.5 e all'art.3). Va ricordato che già la IV Convenzione di Ginevra del 1949, riguardante la protezione dei civili in tempo di guerra, menziona esplicitamente, all'art.27, fra le fattispecie criminali punibili, lo stupro e la prostituzione forzata, ma queste erano considerate condotte criminali solo in quanto azioni tese a ledere il pudore della donna: negli Statuti ICTY e ICTR lo stupro è riconosciuto quale crimine contro l'umanità, alla stregua di fattispecie come assassinio, sterminio, riduzione in schiavitù, deportazione, incarcerazione, tortura e persecuzione per motivi politici, razziali o religiosi, quindi come comportamento dotato di una rilevanza penale autonoma nella cornice del diritto internazionale umanitario.

L'esperienza dei Tribunali ad hoc e della Piattaforma d'Azione adottata dalla IV Conferenza mondiale sulle donne di Pechino (1995), in cui, al punto 11, si sottolinea come «gravi violazioni dei diritti fondamentali delle donne avvengono soprattutto nei periodi di conflitto armato, e producono omicidi, torture, stupri sistematici, gravidanze forzate e aborti forzati, in particolare nelle strategie di "pulizia etnica"», viene fatta propria dalla Corte Penale Internazionale (International Criminal Court - CPI). Nello Statuto della CPI, firmato il 17 luglio 1998, entrato in vigore nel 2002 in esito al deposito del sessantesimo strumento di ratifica, si dispone, all'art.7.g, che sono crimine contro l'umanità «stupro, schiavitù sessuale, prostituzione forzata, gravidanza forzata, sterilizzazione forzata e altre forme di violenza sessuale di analoga gravità»; e, all'art.8.2.XXII, che è crimine di guerra «stuprare, ridurre in schiavitù sessuale, costringere alla prostituzione o alla gravidanza, imporre la sterilizzazione e commettere qualsiasi altra forma di violenza sessuale costituente violazione grave delle Convenzioni di Ginevra». 
L'approccio olistico caratterizza l'azione attuale di contrasto alla violenza nei confronti delle donne degli ultimi anni. A questa nuova visione integrata si deve l'istituzione di UN Women (Entità delle Nazioni Unite per l'uguaglianza di genere e l'empowerment delle donne - United Nations Entity for Gender Equality and the Empowerment of Women - UN Women) sotto l'egida delle NU. UN Women, creata in virtù della ris. 64/289 del 2 luglio del 2010, adottata all'unanimità dall'AG delle NU, nasce dalla fusione di quattro organismi preesistenti: la Divisione per l'Avanzamento delle Donne (Division for the Advancement of Women - DAW), l'Istituto Internazionale di Ricerca e Formazione per l'Avanzamento delle Donne (International Research and Training Institute for the Advancement of Women - INSTRAW), I'Ufficio del Consigliere Speciale sulle Questioni di Genere e l'Avanzamento delle Donne (Office of the Special Adviser on Gender Issues and Advancement of Women - OSAGI) e il Fondo delle Nazioni Unite per lo Sviluppo delle Donne (United Nations Development Fund for Women - UNIFEM). UN Women ha il duplice scopo, da un lato, di supportare gli organismi intergovernativi, a cominciare dalla CSW, per l'elaborazione di politiche, guidelines e norme internazionali; dall'altro, aiutare Stati membri delle NU a implementare gli standards formulati, anche attraverso supporti tecnici e finanziari.

La panoplia delle varie iniziative, lanciate e/o gestite da UN Women per contrastare la violenza nei confronti delle donne è variegata e complessa. Si possono ricordare quali esempi UNiTE to End Violence Against Women, la campagna ideata dal Segretario Generale delle NU nel 2008, volta a sollecitare gli Stati ad adottare e perfezionare le proprie norme nazionali per contrastare il fenomeno della violenza di genere; Say NO - UNiTE to End Violence Against Women, la piattaforma di mobilitazione sociale per la lotta contro la violenza di genere, lanciata nel novembre del 2009 da UN Women e impegnata in azioni di advocacy e sensibilizzazione sul tema; COMMIT, lanciata nel novembre 2012 da UN Women, con lo scopo precipuo di sensibilizzare i leaders politici; I'UN Trust Fund to End Violence Against Women (UN Trust Fund), meccanismo di finanziamento istituito dall'Assemblea Generale delle Nazioni Unite con la risoluzione 50/166 nel 1996 e gestito da UN Women, 
che coopera con organizzazioni non governative e i governi dei Paesi delle Nazioni Unite in primo luogo per prevenire la violenza contro donne e ragazze da parte di gruppi particolarmente a rischio, come adolescenti e donne appartenenti a minoranze indigene o etniche; in secondo luogo per espandere l'accesso delle donne vittime di violenza a servizi, quali l'assistenza legale, la consulenza psicosociale, l'assistenza sanitaria; infine per rafforzare l'attuazione delle leggi, delle politiche e dei piani d'azione sulla violenza di genere, attraverso la raccolta e l'analisi dei dati, al fine di consentire a fornitori di servizi e istituzioni di diventare più efficaci, trasparenti e responsabili.

\section{La tutela delle donne nel quadro regionale}

Parallelamente all'azione condotta a livello globale da parte delle Nazioni unite per contrastare il fenomeno della violenza di genere, si registra la tendenza alla cd regionalizzazione dell'approccio al problema. Molteplici strumenti e convenzioni internazionali, a partire dalla seconda metà degli anni ‘90, sono state infatti adottate in un'ottica, per così dire, glocale, in cui il globale ed il locale, visti come i due lati della stessa medaglia, possono offrire risposte più adeguate, più efficaci e più efficienti perché calibrate ad hoc avuto riguardo alle peculiarità proprie di un contesto, geografico, culturale, sociale, etnico, etc.

È così che il 9 giugno 1994 l'Assemblea generale dell'Organizzazione degli Stati Americani a Belém do Pará, (Brasile) ha adottato la Convenzione inter-americana sulla prevenzione, punizione e sradicamento della violenza contro le donne ${ }^{5}$, entrata in vigore il 5 marzo 1995 di cui sono Stati parti a 32 Paesi del continente americano (GERRERO, 2012, p. 189). In questo Trattato, ai sensi dell'art. 8, gli Stati parti concordano nell'intraprendere progressivamente misure specifiche, compresi programmi, allo scopo di modificare modelli culturali e sociali di comportamento di uomini e donne, compreso lo sviluppo di programmi

5 II testo della Convenzione si legge in http://www.oas.org/juridico/english/treaties/a-61.html 
educativi formali e non formali appropriati ad ogni livello di istruzione, per contrastare i pregiudizi, le consuetudini e tutte le altre prassi basate sull'idea dell'inferiorità o superiorità dell'uno o dell'altro sesso o su ruoli stereotipati per uomini e donne che legittimano o esasperano la violenza contro le donne. Si impegnano a promuovere l'educazione e la formazione di tutte le persone coinvolte nell'amministrazione della giustizia, nella polizia e di tutti gli altri funzionari che si occupano del rispetto della legge, nonché di tutto il personale che attua politiche per la prevenzione, la punizione e lo sradicamento della violenza contro le donne. Gli Stati parte stabiliscono di fornire servizi specializzati adeguati per le donne che hanno subito violenza, attraverso operatori pubblici e privati, compresi rifugi, servizi di consulenza per tutti membri della famiglia se del caso, nonché di cura e custodia per i bambini coinvolti; promuovere e sostenere programmi di educazione a livello governativo o privato finalizzati a sensibilizzare il pubblico riguardo al problema della violenza contro le donne e alle sue soluzioni; incoraggiare i media a sviluppare linee-guida adeguate al fine di contribuire allo sradicamento della violenza contro le donne in tutte le sue forme e rafforzare il rispetto per la dignità delle donne; assicurare la ricerca e la raccolta di statistiche e di informazioni pertinenti circa le cause, le conseguenze e la frequenza delle violenze contro le donne, allo scopo di valutare l'effettività delle misure di prevenzione, sanzione e sradicamento della violenza contro le donne adottate e formulare e attuare gli opportuni cambiamento. Va ricordato altresì che, ex art. 9, rispetto all'adozione delle misure su indicate, gli Stati Parti si obbligano a prendere in particolare considerazione la vulnerabilità alla violenza delle donne in ragione, tra l'altro, della loro razza o origine etnica, della loro condizione di migranti, rifugiate o sfollate. Simile considerazione deve essere prestata alle donne oggetto di violenza durante la gravidanza o alle donne disabili, di minore età, anziane, svantaggiate sul piano socio-economico, coinvolte in conflitti armati o private della libertà. II tallone di Achille di questa Convenzione è la mancata previsione di strumenti concreti di garanzia, che possano fungere sia da deterrente verso atteggiamenti violenti e discriminatori nei confronti delle donne, sia da vera e propria sanzione nei confronti 
dei Paesi parti che vengano meno a quanto statuito, limitandosi a prevedere, all'articolo 12, la possibilità di presentare petizioni individuali ${ }^{6}$ (Salvo quanto si dirà nota 29 infra - ver no artigo original).

Dal canto suo, I'Unione africana ha adottato nel 2003 un Protocollo Addizionale alla Carta africana dei Diritti dell'Uomo, a Maputo in Mozambico, ${ }^{7}$ che, pur non essendo esclusivamente dedicato al tema della violenza di genere, è da ricordare in queste brevi note in quanto, il Protocollo di Maputo contempla una vasta gamma di diritti della donna: l'eliminazione di qualsiasi forma di discriminazione, il diritto alla dignità, alla vita, all'integrità e alla sicurezza fisica della sua persona, la protezione in occasione dei conflitti armati, il diritto all'istruzione e alla formazione, i diritti economici e alla dignità sociale, come pure i diritti alla salute e quelli riproduttivi. Va ricordato l'art. 4, in virtù del quale qualsiasi donna ha diritto al rispetto della sua vita, della sua integrità fisica ed alla sicurezza della sua persona. Ogni tipo di sfruttamento, di punizione e di trattamento inumano o di degrado deve essere vietato. A tal fine gli Stati Membri si impegnano ad adottare misure idonee ed effettive per: adottare e rafforzare le leggi che proibiscono qualsiasi violenza nei confronti delle donne, compresi i rapporti sessuali non desiderati o forzati, che abbiano luogo in privato o in pubblico; adottare qualsiasi altra misura legislativa, amministrativa, sociale, economica e altra per prevenire, reprimere e sradicare qualsiasi forma di violenza nei confronti delle donne; identificare le cause e le conseguenze delle violenze contro le donne e adottare misure idonee per prevenirle ed eliminarle; promuovere attivamente l'istruzione alla pace attraverso programmi d'insegnamento e di comunicazione sociale con l'obiettivo di sradicare gli elementi contenuti nelle credenze e gli atteggiamenti tradizionali e culturali, le pratiche e gli stereotipi che legittimano e inaspriscono la persistenza e la tolleranza della violenza nei confronti delle donne;

Salvo quanto si dirà nota 29 infra - ver no artigo original

II testo del Protocollo si legge in: http://www.au.int/en/content/protocol-african-charter-humanand-peoples-rights-rights-women-africa. In dottrina si veda F. QUILLĖRE-MAJZOUB, Le protocole à la Charte africaine des droits de l'homme et des peuples relatif aux droits de la femme en Afrique: un projet trop ambitieux? Revue trimestrielle des droits de l'homme, 2008, p. 127-162. 
reprimere gli autori della violenza nei confronti delle donne e realizzare programmi in previsione della loro riabilitazione; organizzare meccanismi e servizi accessibili per garantire l'informazione, la riabilitazione e la compensazione effettiva delle donne vittime delle violenze; prevenire e condannare il traffico di donne, perseguire gli autori di questo traffico e proteggere le donne più esposte questo rischio; proibire ogni esperimento medico o scientifico sulle donne senza il loro consenso con cognizione di causa; stanziare risorse di bilancio adeguate per l'attuazione e lo svolgimento di azioni mirate a prevenire e sradicare le violenze contro le donne; assicurarsi che, nei Paesi in cui esiste ancora, la pena di morte non sia applicata nei confronti della donna incinta o che allatta; assicurarsi che le donne e gli uomini usufruiscano di un accesso uguale alle procedure di determinazione dello statuto di profughi e che le donne rifugiate usufruiscano della tutela totale e delle prestazioni garantite nei termini del diritto internazionale dei profughi, ivi compreso il rilascio di documenti d'identità ed altri documenti. Inoltre, l'articolo 5 del Protocollo di Maputo prevede espressamente la condanna e il divieto di qualsiasi tipo di mutilazione genitale femminili, statuendo che gli Stati membri proibiscono e condannano tutte le forme di pratiche nocive che ledono negativamente i diritti umani delle donne e che sono contrarie alle norme internazionali, impegnandosi ad adottare tutte le misure legislative e non per sradicare queste pratiche.

Per quel che concerne il vecchio continente, il tema di violenza di genere è stato regolamentato (poco, in verità) nel quadro tanto dell'Unione europea, quanto del Consiglio d'Europa. L'Unione europea ha avuto un approccio al tema alquanto a macchia di leopardo: si possono ricordare la Risoluzione 2005/2215 del Parlamento europeo sulla situazione delle donne nelle aree di guerra e sul loro ruolo nei processi di ricostruzione nei paesi in situazione di post-conflitto; il programma STOP, attivo sin dal 1997, e il successivo e più incisivo programma DAFNE, che hanno sostenuto l'azione della cd società civile e delle organizzazioni non governative impegnate nella lotta alla violenza nei confronti delle donne in tutte le sue manifestazioni, e nell'assistenza alle vittime; la Risoluzione (2012/2922(RSP) adottata il 6 febbraio 2013 che affronta il problema 
della violenza di genere in un'ottica ampia, non solo limitata alle situazioni di conflitto, partendo dalla constatazione che la violenza contro le donne persiste in tutti i Paesi del mondo come la violazione più diffusa dei diritti umani. Soprattutto va menzionata l'introduzione, con la Direttiva 2011/99/EU approvata da Parlamento e Consiglio, dell'EPO, ovvero l'Ordine di Protezione Europeo, in virtù del quale l'autorità giudiziaria di uno Stato membro, che abbia predisposto per un suo cittadino un ordine di protezione nei confronti di un terzo che attenta alla sua vita o alla sua integrità psicologica o fisica, quindi anche (soprattutto?) in ipotesi di violenza domestica, stalking, minacce, può estendere la tutela prestata alla vittima in modo tale che la protezione garantita dal Paese originario (issuing State) valga anche in un altro Stato membro in cui il cittadino venga a trovarsi (executing State). II regolamento del luglio 2013 ha esteso l'applicazione dell'EPO dalle sole questioni criminali a quelle civili, assicurando così una sorta di tutela senza frontiere posto che le vittime di violenza domestica possono fare ora affidamento su ordini di restrizione validi a livello dell'Unione nel suo intero territorio ${ }^{8}$.

Un approccio più sistematico è quello che contraddistingue l'azione del Consiglio d'Europa. In verità, la Convenzione Europea per la Salvaguardia dei Diritti dell'Uomo e delle Libertà Fondamentali (CEDU) del 1950, contiene solo poche clausole espressamente dirette alla tutela dei diritti delle donne, in particolare sul diritto matrimoniale e su un generale divieto di discriminazione ${ }^{9}$. Peraltro, in anni recenti, il Consiglio

8 L'Ordine di protezione europeo è stato introdotto nell'ordinamento italiano col decreto legislativo 11.02.2015 n ${ }^{\circ}$ 9, G.U. 23.02.2015, con un po' di ritardo quindi, rispetto alla dead line per il recepimento della direttiva fissata all'11 gennaio 2015. L'art. 4 del decreto introduce, all'interno dell'art. 282-quater c.p.p., un nuovo comma 1-bis mediante il quale si prevede che la persona offesa sia informata della possibilità di richiedere l'emissione di un ordine di protezione europeo, quest'ultimo decretato dal giudice che dispone una delle misure cautelari di cui agli artt. 282-bis c.p.p. (Allontanamento dalla casa familiare) e 282-ter c.p.p. (Divieto di avvicinamento ai luoghi frequentati dalla persona offesa). In questi casi il giudice provvede «su richiesta della persona protetta che dichiari di soggiornare o risiedere all'interno di uno Stato membro ovvero che manifesti l'intenzione di risiedere o soggiornare in altro Stato membro».

9 In effetti, la tutela delle donne ha beneficiato di un'interpretazione evolutiva degli artt. 2, 3, 8 e 14 della Convenzione europea per la salvaguardia dei diritti umani e delle libertà fondamentali che ha posto in essere la Corte di Strasburgo: A. VIVIANI, La violenza contro le donne nell'interpretazione della Corte di Strasburgo, in Diritti umani e diritto internazionale, 2010, p. 412-422., e B. ANCEL, Les violences conjugales saisies par le droit européen: évolution ou révolution? Revue trimestrielle de droit européen, 2013, p. 701-710 
d'Europa si è contraddistinto per un'azione strutturata in tema di violenza di genere che prende le mosse dalla Raccomandazione $\operatorname{Rec}(2002) 5$ del Comitato dei Ministri agli Stati membri sulla protezione delle donne dalla violenza adottata nel 2002. Ai sensi della Raccomandazione il termine "violenza contro le donne" designa qualsiasi azione di violenza fondata sull'appartenenza sessuale che comporta o potrebbe comportare per le donne che ne sono bersaglio danni o sofferenze di natura fisica, sessuale o psicologica, ivi compresa la minaccia di mettere in atto simili azioni, la costrizione, la privazione arbitraria della libertà, sia nella vita pubblica che in quella privata. Questa definizione si applica, ma non è circoscritta, alle seguenti azioni: violenza perpetrata all'interno della famiglia o delle mura domestiche e in particolare le aggressioni di natura fisica o psichica, gli abusi di tipo emotivo o psicologico, lo stupro e l'abuso sessuale, l'incesto, lo stupro fra coniugi, partner abituali, partner occasionali o conviventi, i crimini commessi in nome dell'onore, la mutilazione degli organi genitali o sessuali femminili, così come le altre pratiche tradizionali dannose per le donne, quali i matrimoni forzati; violenza perpetrata nella comunità in generale e in particolare lo stupro, gli abusi, le molestie sessuali e le intimidazioni sul luogo di lavoro, nelle istituzioni o in altri luoghi, la tratta delle donne a fini di sfruttamento sessuale; violenza perpetrata o tollerata dallo Stato o dagli agenti della forza pubblica; la violazione dei diritti fondamentali delle donne in situazione di conflitto armato, in particolare la presa di ostaggi, la deportazione, lo stupro sistematico, la schiavitù sessuale, la gravidanza forzata e la tratta ai fini di sfruttamento sessuale ed economico.

Una definizione così sfaccettata offre poche vie di fuga, maglie così strette sono in grado di imbrigliare azioni anche non sfacciatamente ascrivibili alla violenza di genere: ma è uno strumento di soft law e, come tale, non comporta obblighi per gli Stati cui è rivolta, poiché il suo valore è eminentemente esortativo. Vero è che la Raccomandazione ha costituito sia la base per una campagna multilivello (intergovernativo, parlamentare e locale) fra i Paesi membri del Consiglio d'Europa; sia il trampolino di lancio per la Convenzione di Istanbul. 
Sotto il primo profilo, in base al Piano d'azione adottato a conclusione del Terzo Vertice dei Capi di Stato e di governo degli Stati membri del Consiglio d'Europa, a partire dal 2006 è stata condotta una campagna suddivisa in quattro grandi filoni - misure politiche e legali, supporto e protezione per le vittime, raccolta di dati e azioni di informazione - volta a sensibilizzare l'opinione pubblica e indurre gli Stati a conformarsi a quanto disposto nella Raccomandazione, attraverso l'adozione di misure efficaci e efficienti.

Sotto il secondo profilo, in esito al Rapporto finale della Task Force istituita per sostenere e gestire tale campagna (COUNCIL OF EUROPE, online, 2008) Rapporto che raccomandava l'adozione da parte del Consiglio d'Europa di uno strumento complessivo giuridicamente vincolante in materia di diritti umani, al fine di prevenire e combattere ogni forma di violenza sulle donne, il Comitato dei Ministri ha istituito nel dicembre 2008 un gruppo di esperti (Comitato Ad Hoc per prevenire e combattere la violenza contro le donne e la violenza domestica CAHVIO) con lo scopo specifico di redigere un draft di trattato in tema di contrasto alla violenza di genere. II CAHVIO ha presentato nel giugno 2009 un Interim Report, in cui erano esposti contenuti e metodologia della bozza proposta. Dopo la sua approvazione, il documento ha costituito la base di una serie di incontri fra il CAHVIO, delegati degli Stati membri e rappresentanti della cd società civile, che hanno avuto luogo tra febbraio e dicembre 2010. Infine, il testo è stato approvato e trasmesso al Comitato dei Ministri per essere portato all'attenzione dell'Assemblea Parlamentare, nel marzo 2011. II Comitato dei Ministri ha quindi adottato il testo il 7 aprile 2011 sicché la Convenzione è stata aperta alla firma in occasione della Conferenza ministeriale di Istanbul l'11 maggio 2011.

\section{La Convenzione di Istanbul}

La Convenzione sulla prevenzione e la lotta contro la violenza nei confronti delle donne e la violenza domestica è stata adottata quindi dal Comitato dei Ministri del Consiglio d'Europa il 7 aprile 2011 ed è stata 
aperta alla firma l'11 maggio 2011 in occasione della $121^{\mathrm{ma}}$ sessione del Comitato dei Ministri a Istanbul. II deposito degli strumenti di ratifica di Andorra e Danimarca, avvenuto rispettivamente il 22 e 23 aprile 2014, ha consentito alla Convenzione di entrare in vigore lo scorso $1^{\circ}$ agosto, ai sensi di quanto stabilito dall'art. 75, par. $3 .^{10}$ La Convenzione è aperta alla firma degli Stati membri del Consiglio d'Europa, degli Stati non membri che hanno partecipato alla sua elaborazione (Canada, Giappone, Messico, Santa Sede, Stati Uniti) e dell'Unione Europea ${ }^{11}$.

La Convenzione contiene 81 articoli divisi in 12 capitoli, la cui struttura riposa su quattro pilastri, che potremmo definire delle "4P": prevenzione, protezione, procedure, politiche integrate.

Sotto il primo profilo, la Convenzione sottolinea come, nel contrasto alla violenza di genere, la prevenzione sia di primaria importanza. A

10 L'art. 75, par. 3, della Convenzione individua l'entrata in vigore di quest'ultima nel primo giorno del mese successivo alla scadenza di un periodo di tre mesi dopo la data in cui dieci firmatari, di cui almeno otto Stati membri del Consiglio d'Europa, abbiano espresso il loro consenso ad essere vincolati. Nell'ordine, hanno ratificato: Turchia (1.3.2012), Albania (4.2.2013), Portogallo (5.2.2013), Montenegro (22.4.2013), Italia (10.9.2013), Bosnia Erzegovina (7.11.2013), Austria (14.11.2013), Serbia (21.11.2013), Spagna (10.4.2014), Andorra (22.4.2014), Danimarca (23.4.2014), Svezia (1.7.2014), Francia (4.7.2014), Malta (29.7.2014), Monaco (7.10.2014), Slovenia (5.2.2015). In particolare, l'Italia ha firmato la Convenzione di Istanbul il 27 settembre 2011; la Camera dei Deputati ha approvato all'unanimità la ratifica della Convenzione il 28 maggio 2013 e il Senato, sempre all'unanimità, il 19 giugno 2013, sicché con legge n. 77 del 27 giugno 2013, il Parlamento ha poi autorizzato il Presidente della Repubblica a ratificare tale Convenzione e ne ha contestualmente emesso l'ordine di esecuzione. Secondo quanto prescritto dall'art. 75 , par. 2, della Convenzione, lo strumento di ratifica italiano è stato quindi depositato presso il Segretariato generale del Consiglio d'Europa il 10 settembre 2013.

11 Sarebbe interessante, ma non è consentito dallo spazio a disposizione di queste brevi note, l'indagine della ancora in fieri adesione dell'Unione europea alla Convenzione di Istanbul, in quanto si tratterebbe del secondo caso di prassi (finora l'Unione è parte solamente di un unico trattato sui diritti umani, ossia la Convenzione delle Nazioni Unite sulle persone disabili). L'opportunità di perfezionare la propria partecipazione alla Convenzione di Istanbul da parte dell'Unione è evidente laddove si pensi al fatto che ciò consentirebbe di prevenire criticità che altrimenti deriverebbero dal c.d. double standard, spesso praticato in materia di diritti della persona umana. L'Unione, infatti, è solita chiedere agli Stati aspiranti membri o associati di garantire i diritti garantiti da trattati internazionali di cui essa non è però parte contraente. In effetti, solitamente si tratta di accordi sui diritti umani non aperti all'adesione dell'Unione. Nel caso di specie, l'Unione è invece abilitata a partecipare alla Convenzione di Istanbul e, pertanto, se essa per prima non agisse in questa direzione, potrebbe poi trovarsi in difficoltà nel richiedere il rispetto dei diritti garantiti da quella stessa Convenzione agli Stati. Si veda in dottrina S. PEERS, Should the EU Ratify the Istanbul Convention on Violence against Women? 23 aprile 2014. Disponível em: <eulawanalysis. blogspot.it>. Acesso em: 03 out. 2015. 
tal fine, gli Stati e i governi che hanno ratificato la Convenzione sono ora obbligati ad adottare le seguenti misure: formare il personale che è a stretto contatto con le vittime; adottare regolarmente campagne di sensibilizzazione; includere nel materiale pedagogico argomenti quali la parità tra i sessi e la risoluzione non violenta dei conflitti nelle relazioni interpersonali; predisporre programmi terapeutici per gli autori di violenza domestica e per i delinquenti sessuali; lavorare strettamente con le organizzazioni non governative; coinvolgere i media e il settore privato affinché siano eliminati gli stereotipi di genere e sia promosso il mutuo rispetto.

Sotto il secondo profilo, rileva la protezione che, in occasione di episodi di violenza, dev'essere garantita alle vittime e ai testimoni. Ciò comporta l'intervento e la tutela da parte delle forze di polizia e dei servizi d'aiuto specializzati. Le azioni concrete che la Convenzione indica per rispondere con efficacia a questa esigenza sono molteplici, fra cui ricordiamo: dare alle forze di polizia il potere di allontanare l'autore di violenza domestica dal suo domicilio, poiché in caso di pericolo immediato, la polizia deve poter garantire la sicurezza della vittima; assicurare alle vittime l'accesso a informazioni utili, in modo che le stesse possano comprendere facilmente i servizi e gli aiuti che sono a loro disposizione; distribuire sul territorio dei luoghi protetti accessibili e in numero sufficiente; fornire un'assistenza telefonica specializzata e gratuita a livello nazionale per 24 ore al giorno, 7 giorni la settimana; creare dei centri di crisi facilmente accessibili per le vittime di stupro e violenza sessuale; tali centri dovranno fornire assistenza e consulenza medica immediata e assicurare assistenza medico-legale; tener presente che non è sufficiente porre in essere delle strutture di protezione e dei servizi di aiuto e assistenza, ma è anche necessario garantire che le vittime siano informate dei loro diritti e che le stesse sappiano dove e come ottenere aiuto.

Sotto il terzo profilo, le procedure da seguire in caso di violenza di genere, la Convenzione di Istanbul ne definisce e qualifica penalmente diverse forme. Ciò implica che gli Stati parte devono, se del caso, introdurre nel loro sistema nazionale nuove figure di reato, tra cui: la 
violenza psicologica e psichica, la violenza sessuale e lo stupro, la persecuzione, le mutilazioni genitali femminili, il matrimonio forzato, l'interruzione di gravidanza e la sterilizzazione forzate ${ }^{12}$. Ancora, gli Stati aderenti dovranno fare in modo che attenuanti o le giustificazioni che collegano la violenza commessa a fattori culturali o a tradizioni legate all'onore, siano eliminate. La tipizzazione di queste figurae criminis comporta che gli Stati dovranno adottare una serie di misure affinché vi siano indagini effettive per ogni denuncia di violenza. Le autorità inquirenti e le forze di polizia dovranno rispondere alle richieste di aiuto, raccogliere le prove e valutare il rischio di violenza per proteggere adeguatamente la vittima, garantendo altresì che i diritti delle vittime siano rispettati in tutti gli stadi della procedura e che sia a loro evitata ogni forma di vittimizzazione secondaria.

Sotto il quarto profilo, la Convenzione sottolinea l'importanza di politiche integrate alla luce della considerazione che una sola autorità statale non sarebbe in grado di fronteggiare efficacemente la violenza nei confronti delle donne e alla violenza domestica. Per dare una risposta efficace a questo tipo di violenze, è indispensabile un'azione concertata da parte di numerosi soggetti coinvolti, sicché la Convenzione chiede agli Stati di porre in essere delle politiche globali e coordinate che coinvolgano gli organismi pubblici, le organizzazioni non governative, i parlamenti e le autorità nazionali, regionali e locali. L'idea è quella di istituire un sistema integrato top-down, attraverso la formulazione di un piano di intervento nazionale che individui missione e ruolo di ciascun stakeholder coinvolto nel contrasto alla violenza di genere.

II sistema delle 4P si completa con un meccanismo di controllo, destinato a valutare in che misura le disposizioni della Convenzione

\footnotetext{
12 Per esempio, per quel che concerne il nostro ordinamento, è ipotizzabile che debba essere emendato l'art. 18 della legge n. 194 del 22 maggio 1978 in cui si parla di aborto "cagionato" o "provocato", senza mai usare l'espressione "aborto forzato", inserita invece nell'art. 39, lett. a), della Convenzione di Istanbul. Ai sensi del rapporto esplicativo allegato alla Convenzione, questa seconda locuzione sembra assumere un'accezione diversa, e ben più grave, rispetto alle fattispecie disciplinata dalla legge italiana.
} 
sono applicate a livello di ciascun Stato parte. Tale meccanismo si basa su due organismi, il Gruppo di esperti per la lotta contro la violenza nei confronti delle donne e la violenza domestica (Group of Experts on Violence - GREVIO), costituito da esperti indipendenti, e il Comitato delle Parti, organo politico composto da rappresentanti ufficiali degli Stati che hanno ratificato la Convenzione. II Comitato delle Parti elegge i membri del GREVIO e trasmette agli Stati aderenti raccomandazioni aventi a oggetto le misure necessarie per eseguire le indicazioni del GREVIO. II GREVIO è deputato a ricevere ed esaminare le relazioni periodiche degli Stati parte e di organizzazioni non governative, istituzioni nazionali per i diritti umani e rappresentanti della società civile e, su queste basi, valuta le misure adottate dagli Stati per dare attuazione alla Convenzione. II GREVIO può inoltre effettuare delle ispezioni in loco ad hoc per valutare e indagare su una specifica questione. In esito a tale procedura di controllo, il GREVIO può adottare dei Rapporti in cui indicare conclusioni in grado di aiutare il singolo Stato contraente a migliorare l'applicazione e il rispetto della Convenzione. Alla pubblicazione del Rapporto del GREVIO possono seguire Raccomandazioni formulate dal Comitato delle Parti, ma tali Raccomandazioni non sono obbligatorie, né nell'essere formulate, né nel loro contenuto.

\section{Conclusione}

La Convenzione di Istanbul rappresenta sicuramente un punto di riferimento a livello internazionale per quanto riguarda la lotta alla violenza contro le donne, secondo le parole del Vice Direttore Esecutivo di UN Women, John Hendra, nel corso di un evento nella giornata di apertura della 56 ${ }^{\mathrm{ma}}$ sessione della CSW nel 2012 (UNITED NATIONS, online, 2012), come pure una pietra miliare del sistema di tutela dei diritti umani, come dichiarato dal Presidente dell'Assemblea parlamentare del Consiglio d'Europa Anne Brasseur (COUNCIL OS EUROPE, online, 2014). Peraltro, non bisogna sottacere alcune criticità che si vogliono brevemente evidenziare in queste osservazioni conclusive. 
In primo luogo, sotto il profilo ratione materiae, la Convenzione patisce di una serie di riserve ${ }^{13}$, che hanno avuto come prodromi tentativi di ridurre la portata, attraverso un'applicazione restrittiva, della violenza di genere. Per esempio, Federazione Russa e Santa Sede hanno proposto di escludere la violenza contro lesbiche, bisessuali e transgender dall'ambito di applicazione del trattato (sancita nell'art 4). II Regno Unito si è opposto alla penalizzazione del matrimonio forzato, sancita dalla Convenzione nell'art. 37 e ha altresì proposto l'esclusione dall'ambito di applicazione delle violenze perpetrate nel corso di conflitti armati e il non inserimento della violenza contro le donne tra le violazioni dei diritti umani (sancita nell'art.3), nonostante si tratti, in entrambi i casi, di estensioni ormai unanimemente recepite nei più recenti strumenti internazionali adottati in materia, alcuni dei quali abbiamo espressamente visto nei paragrafi precedenti di queste brevi note ${ }^{14}$. II nostro Paese ha avuto un atteggiamento alquanto ambiguo, quanto all'estensione delle garanzie. In effetti, in occasione del primo disegno di legge di ratifica della Convenzione di Istanbul, presentato dal Governo nel gennaio 2013, il Rappresentante italiano permanente presso il Consiglio d'Europa ha depositato una dichiarazione interpretativa in lingua inglese del seguente tenore: «ltaly declares that it will apply the Convention in conformity with the principles and provisions of the Italian Constitution» ossia l'Italia applicherà la Convenzione nel rispetto dei princìpi e delle previsioni costituzionali. Questa dichiarazione, posta in essere quale escamotage per aggirare il divieto di riserve sancito dalla

13 II testo completo delle riserve e delle dichiarazioni interpretative si legge in: http://conventions. coe.int/Treaty/Commun/ListeDeclarations.asp?NT=210\&CM=1\&DF=\&CL=ITA\&VL=1

14 In verità, anzi, dalla ricostruzione storica qui proposta, sembra che dalla Convenzione CEDAW, starting point degli strumenti internazionali vincolanti aventi a oggetto la tutela delle donne, dall'impianto concettuale di stampo prevalentemente emancipatorio, sia proprio attraverso gli interventi emergenziali su "donne nei conflitti" attuati nel contesto di scenari di guerra a partire dagli anni ' 90 , che si sia approdati alla consapevolezza che la questione della violenza di genere ha una valenza politica a livello globale. 
Convenzione, salvo che per clausole espressamente indicate ${ }^{15}$, sembra riconducibile al carattere troppo ampio e incerto attribuito da talune norme convenzionali alla nozione di "genere", ritenuta quindi potenzialmente incompatibile con l'ordinamento italiano ${ }^{16}$. Soprattutto alla luce della dizione dell'art. 3, lett. c), secondo cui con tale nozione deve intendersi «the socially constructed roles, behaviours, activities and attributes that a given society considers appropriate for women and men» («con il termine "genere" ci si riferisce a ruoli, comportamenti, attività e attributi socialmente costruiti che una determinata società considera appropriati per donne e uomini»), la dichiarazione è stata posta in essere al fine di restringere l'applicazione dell'accordo, dai confini troppo ampi e fumosi, probabilmente a detrimento dei transessuali ${ }^{17}$.

15 L'art. 78 sancisce che: «1. Non è ammessa alcuna riserva alle disposizioni della presente Convenzione, salvo quelle previste ai successivi paragrafi 2 e 3; 2. Ogni Stato o I'Unione europea può, al momento della firma o del deposito del proprio strumento di ratifica, di accettazione, di approvazione o di adesione, mediante dichiarazione inviata al Segretario Generale del Consiglio d'Europa, precisare che si riserva il diritto di non applicare o di applicare solo in particolari casi o circostanze le disposizioni enunciate nei seguenti articoli: art. 30.2 (risarcimento); art. 44. 1, 3,4 (giurisdizione); art. 55,1 esaminato insieme all'art.35 per quanto riguarda i reati minori (Procedimenti d'ufficio ed ex parte); art. 58 esaminato insieme agli art. 37, 38 e 39 (prescrizione); Art. 59 (status di residente). 3. Ogni Stato o l'Unione europea può, al momento della firma o del deposito dello strumento di ratifica, di accettazione, di approvazione o di adesione, mediante dichiarazione inviata al Segretario Generale del Consiglio d'Europa, precisare che si riserva il diritto di prevedere sanzioni non penali, invece di imporre sanzioni penali, per i comportamenti di cui agli articoli 33 (violenza psicologica) e 34 (violenza fisica). 4. Ogni Parte può ritirare in tutto o in parte una riserva mediante notifica indirizzata al Segretario Generale del Consiglio d'Europa. II ritiro avrà effetto a partire dalla data del suo ricevimento da parte del Segretario Generale».

16 Il documento, Disegno di legge n. 3654 dell'8 gennaio 2013, Ratifica ed esecuzione della Convenzione del Consiglio d'Europa sulla prevenzione e la lotta contro la violenza nei confronti delle donne e la violenza domestica, fatta a Istanbul l'11 maggio 2011, p. 7, presentato dall'allora Ministro degli Affari esteri, Giulio Terzi di Sant'Agata, e dal Ministro del Lavoro e delle Politiche sociali con delega alle Pari opportunità, Elsa Fornero, si legge online sul sito ufficiale del Senato della Repubblica: www.senato.it

17 Ciò lascia perplessi alla luce di due considerazioni, una di matrice interna e una di stampo internazionalistico. Sotto il primo profilo, l'art. 3.1 della nostra Costituzione sancisce il principio di uguaglianza formale, in virtù della quale si vieta espressamente che possano essere previsti trattamenti differenziati a causa di uno dei motivi da essa elencati, tra cui anche il divieto di discriminazioni per motivi di sesso, che si riflette anche sulla posizione dei transessuali e degli omosessuali. Sotto il secondo profilo, benché la dizione letterale della denominazione ufficiale della Convenzione di Istanbul mira espressamente a salvaguardare (principalmente) le donne, il suo scopo è quello di incoraggiare gli Stati parti ad agire a tutela di ogni vittima di violenza domestica (art. 2, par. 2), evidentemente senza alcuna contrapposizione di sesso. 
Tuttavia, anche a seguito delle elezioni politiche del febbraio 2013, è stata presentata una nuova proposta di legge di ratifica non contenente alcun cenno alla dichiarazione interpretativa in parola. Questa seconda proposta è stata poi adottata, consentendo la promulgazione della legge di ratifica e il ritiro, il 12 settembre 2013 (ovvero due giorni dopo il deposito dello strumento di ratifica), della dichiarazione interpretativa italiana ex art. 78, par. 4, della Convenzione, il che, se alla luce delle considerazioni critiche appena esposte va salutato con plauso, pur nondimeno dà contezza e visibilità di atteggiamenti poco lineari del nostro Governo.

Sempre nel quadro dei limiti riscontrabili nella Convenzione, va sottolineato come alcuni Stati firmatari abbiano presentato proposte miranti a cancellare il principio della "dovuta diligenza" da parte degli Stati nel prevenire, indagare, punire i responsabili e risarcire le vittime di atti di violenza (art 5), o a indebolire il previsto obbligo da parte degli Stati di favorire e agire da mediatori nelle eventuali azioni individuali o collettive condotte nell'ambito dei meccanismi regionali e internazionali di denuncia delle violenze (art 21). In tal senso si è espressa la Federazione Russa, mentre il Regno Unito ha avanzato proposte volte a limitare le misure attive finalizzate a favorire la formulazione di politiche e linee guida di concerto con i media, proponendo invece un generico incoraggiamento ai media per indurli a modificare politiche e strategie comunicative (art 17). Queste proposte, in parte rifiutate, hanno come conseguenza un infausto rallentamento nel processo di ratifica da parte di tutti gli Stati membri del Consiglio d'Europa.

Ancora, può evidenziarsi un terzo nodo critico riguarda le procedure di monitoraggio e garanzia stabilite dalla Convenzione, sia per proposte di emendamento - presentate in particolare dalla Federazione Russa - relative alla pubblicazione di rapporti e missioni in loco da parte del GREVIO (art. 68) e alle riserve (previste in merito ad alcuni articoli specifici della Convenzione) che devono essere precisate dalle parti contraenti al momento della firma o del deposito dello strumento di ratifica, esponendo i motivi (art. 79); sia perché il meccanismo di controllo illustrato supra ha due evidenti limiti. Il primo è quello di non essere aperto a ricorsi di singoli. Infatti, la Convenzione non attribuisce né al GREVIO né al Comitato 
delle Parti competenza a ricevere comunicazioni individuali o interstatali riguardanti l'eventuale violazione delle sue disposizioni ${ }^{18}$. II secondo riguarda le Raccomandazioni del Comitato delle Parti sia perché, come detto, l'adozione delle raccomandazioni non è obbligatoria, sia perché dipendono dalla volontà di un organismo composto da Stati e non da personalità indipendenti.

Infine un quarto gruppo di criticità riguarda la tutela, nel quadro della Convenzione, delle donne migranti o rifugiate: in particolare, il previsto impegno da parte degli Stati membri a prendere misure legislative o di altro tipo allo scopo di garantire che la violenza contro le donne basata sul genere possa essere riconosciuta come una forma di persecuzione ai sensi dell'articolo $1 \mathrm{~A}$ (2) della Convenzione relativa allo Status dei Rifugiati del 1951 e come una forma di grave pregiudizio che dia luogo a una protezione complementare (art 60). La Federazione Russa ha contestato tale previsione e ha proposto un emendamento contemplante una lista esaustiva di particolari crimini che, se accolta, avrebbe avuto il probabile effetto di limitare ulteriormente le forme di violenza che permettano alle donne di godere in maniera piena del diritto di asilo. Rispetto al diritto di non refoulement, previsto dall'art. 61, è stato proposto dal Regno Unito, e non accolto, un emendamento mirante a limitare tale diritto ai soli casi in cui fosse ravvisabile e verificabile un "reale pericolo" per le richiedenti asilo. L'Italia ha proposto un'aggiunta al paragrafo 4 dell'art. 59, allo scopo di subordinare il diritto di residenza delle vittime di matrimonio coatto all'avvenuto scioglimento o annullamento del matrimonio che ha determinato la decadenza di tale diritto: è evidente come ciò comporti il rischio di negare un intervento

18 Sotto questo profilo quindi offre minori garanzie degli analoghi strumenti interamericano ed africano. La Convenzione di Belém do Parà, infatti, stabilisce che individui e organizzazioni non governative possono inoltrare petizioni alla Commissione interamericana dei diritti dell'uomo (art. 12); inoltre, agli Stati e alla Commissione interamericana per le donne è riconosciuta la possibilità di adire in via consultiva anche la Corte interamericana per questioni attinenti l'interpretazione della Convenzione (art. 11). II Protocollo di Maputo predispone un sistema di rapporti periodici da inviare alla Commissione africana dei diritti dell'uomo e dei popoli, il che si affianca alla possibilità che di casi concernenti violazioni dei diritti da esso garantiti venga investita la Corte africana a fronte dell'ampia competenza ratione materiae che ad essa è conferita dagli artt. 3 e 7 del suo Statuto. 
efficace e pronto nella protezione delle donne soggette a matrimoni forzati dato lo iato temporale fra l'avvio della procedura di annullamento e divorzio e l'esito finale.

In linea generale, dall'analisi delle disposizioni della Convenzione emerge sì un approccio olistico al problema della violenza nei confronti delle donne che però evidenzia una sostanziale ottica emergenziale, posto che non ci sia il necessario spazio dedicato a considerazioni relative all'importanza che i fattori socioeconomici e culturali hanno nel determinare il fenomeno, salvo poche norme specificamente indirizzate alla lotta ai fattori culturali alla base della discriminazione e della violenza. In secondo luogo, la violenza nei confronti delle donne è nella Convenzione, per così dire, "normalizzata": ossia viene resa un paradigma interpretativo nell'ambito non solo delle relazioni tra i generi, ma di tutte le relazioni di diversità nei confronti di soggetti vulnerabili (anziani, bambini, migranti) particolarmente esposti alla violenza domestica, il che oltre a differenziarsi dal tradizionale approccio dei movimenti di emancipazione e liberazione femminile, va in parte a detrimento della specificità e unicità della discriminazione di genere. Da questa "normalizzazione" deriva l'ulteriore conseguenza di non condividere l'impianto emancipatorio proprio della CEDAW che nelle differenze di genere vede ostacoli da rimuovere e/o superare, ma, al contrario, li acquisisce quali dati di fatto, ciò che potrebbe determinare nella prassi una minore attenzione ai fattori di prevenzione, dando prevalenza alla tutela delle vittime e al perseguimento di coloro che commettono violenza.

Queste considerazioni critiche non devono essere intese nel senso di sminuire la portata della Convenzione di Istanbul ma solo ribadire l'assunto espresso mirabilmente da Friedrich Hacker «la violenza è semplice; le alternative alla violenza sono complesse».

\section{Referências}

AMERICAN ASSOCIATION OF UNIVERSITY WOMEN. Hostile Hallways: Bullying, Teasing, and Sexual Harassment in School. 2001. 
Disponível em: <http://www.aauw.org/files/2013/02/hostile-hallwaysbullying-teasing-and-sexual-harassment-in-school.pdf>. Acesso em: 20 ago. 2015.

AMNESTY INTERNATIONAL. 2004. Disponível em: < http://www. amnesty.org/en/library/asset/AFR47/007/2004/en/53d74ceb-d5f7-11ddbb24-1fb85fe8fa05/afr470072004en.pdf>. Acesso em 09 set. 2015.

BROUWER, Anne-Marie. Supranational Criminal Prosecution of Sexual Violence. Intersentia: Oxford, 2005.

COUNCIL OF EUROPE. Council of Europe Convention on preventing and combating violence against women and domestic violence. 2011. Disponível em: <http://www.conventions.coe.int/Treaty/ITA/ Treaties/Html/210.htm> Acesso em: 12 set. 2015

COUNCIL OF EUROPE. Final Activity Report, Task Force to Combat Violence against Women. 2008. Disponível em: < http://www.coe.int/t/ dg2/equality/domesticviolencecampaign/Source/Final_Activity_Report. pdf>. Acesso em: 02 set. 2015.

COUNCIL OF EUROPE. Parliamentary Assembly Assemblée Parlamentaire. Istanbul Convention: putting an end to the cycle of violence against women. 2014. Disponpivel em: <http://assembly.coe.int/nw/xml/News/News-View-EN. asp ? newsid=5313\&lang=2\&cat $=135>$ Acesso em: 11 set. 2015

WORLD HEALTH ORGANIZATION. Global and Regional Estimates of Violence against Women. 2013. Disponível em: < http://apps.who. int/iris/bitstream/10665/85239/1/9789241564625_eng.pdf>. Acesso em 20 ago. 2015

UNICEF. Hidden in Plain Sight: A Statistical Analysis of Violence against Children. 2014a. Disponível em: <http://www.unicef. org/publications/files/Hidden_in_plain_sight_statistical_analysis_ Summary_EN_2_Sept_2014.pdf>. Acesso em: 23 jun. 2015.

GUERRERO, P. Mejía. La Comisión interamericana de mujeres y la Convención de Belém do Parà, in Revista interamericana y europea de 
derechos humanos. Int.Am. \& Eur.Hum.Rts.J., [s.I], v. 56, 2012, p. 189213.

HUMANS RIGHT WATCH. Bosnia: Landmark Verdicts for Rape, Torture and Sexual Enslavement. 2001. Disponível em: <https://www. hrw.org/news/2001/02/22/bosnia-landmark-verdicts-rape-torture-andsexual-enslavement>. Acesso em: 02 abr. 2015

UNITED NATIONS. In-depth Study on All Forms of Violence against Women: Report of the Secretary-General. 2006. Disponível em: <http://daccess-dds-ny.un.org/doc/UNDOC/GEN/N06/419/74/PDF/ N0641974.pdf?OpenElement>. Acesso em: 20 ago. 2015.

INTERNATIONAL LABOUR ORGANIZATION. Global estimate of forced labour: results and methodology. 2012. Disponível em: <http://www.ilo.org/wcmsp5/groups/public/---ed_norm/---declaration/ documents/publication/wcms_182004.pdf>. Acesso em: 14 set. 2015.

UNICEF. Female Genital Mutilation/Cutting: what might the future hold. 2014b. Disponível em: <http://www.unicef.org/media/files/FGM-C_ Report_7_15_Final_LR.pdf>. Acesso em: 02 set. 2015.

UNITED NATIONS. Convention on the Elimination of All Forms of Discrimination against Women New York. 1979. Disponível em: <http:// www.ohchr.org/EN/Professionallnterest/Pages/CEDAW.aspx>. Acesso em: 02 out. 2015

UNITED NATIONS. UN Women supports the Council of Europe's Convention against Domestic Violence. 2012. Disponível em: <http:// www.unwomen.org/en/news/stories/2012/2/un-women-supports-thecouncil-of-europe-s-convention-against-domestic-violence>. Acesso em: 12 set. 2015

Recebido em: 23/11/2015

Aprovado em: 02/12/2015 